Archive for

Organic Chemistry

Arkivoc 2018, part iii, 153-164

\title{
Water-dependent synthesis of biologically active diaryl diselenides
}

\author{
Agata J. Pacuła ${ }^{a}$, Magdalena Obieziurska a , Jacek Ścianowski ${ }^{* a}$, Katarzyna B. Kaczorb, \\ and Jędrzej Antosiewicz ${ }^{\text {b,c }}$ \\ ${ }^{a}$ Department of Organic Chemistry, Faculty of Chemistry, Nicolaus Copernicus University in Torun, \\ 7 Gagarin Street, 87-100 Torun, Poland \\ ${ }^{b}$ Department of Bioenergetics and Physiology of Exercise, Medical University of Gdansk, \\ 1 Debinki Street, 80-211 Gdansk, Poland; ' Department of Biochemistry, Gdansk University of Physical Education \\ and Sport, 1 Kazimierza Gorskiego Street,, 80-336 Gdansk, Poland \\ E-mail: jsch@chem.umk.pl
}

Received 08-23-2017

Accepted 11-30-2017

Published on line $02-18-2018$

\section{Abstract}

A new one-step method for the synthesis of diaryl diselenides has been developed. The reaction of 0 iodobenzamides with dilithium diselenide can be controlled by the presence of water providing a simple and efficient protocol to obtain benzisoselenazolones or diaryl diselenides. A series of $\mathrm{N}$-aryl ebselen derivatives and the corresponding diselenides was obtained. All synthesized compounds were tested in vitro as antioxidants and cytotoxic agents. $\mathrm{N}$-(2,3,4-trimethoxyphenyl)benzisoselenazol-3(2H)-one was the best in vitro antioxidant and the corresponding diselenide the most potent cytotoxic agent against prostate cancer cell line DU145, being inactive towards healthy prostate cell line PNT1A.
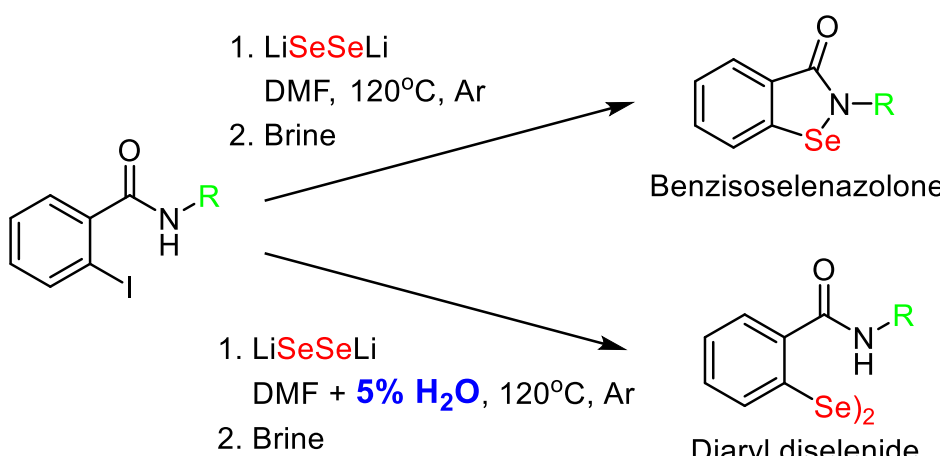

$$
\begin{aligned}
& \mathrm{R}=-\mathrm{C}_{6} \mathrm{H}_{4}-\mathrm{OCH}_{3} \\
& \text { antioxidant } \\
& \text { activity } \\
& \mathrm{R}=-\mathrm{C}_{6} \mathrm{H}_{4}-4-\mathrm{OCH}_{3} \\
& \mathrm{R}=-\mathrm{C}_{6} \mathrm{H}_{2}-2,3,4-\left(\mathrm{OCH}_{3}\right)_{3} \\
& \text { cytotoxic }
\end{aligned}
$$

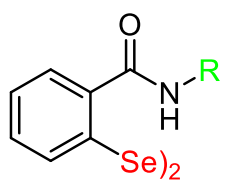

Diaryl diselenide activity

Keywords: Benzisoselenazolones, diselenides, selenium, cytotoxicity, antioxidants, biological activity 


\section{Introduction}

Selenium-selenium bond is a unique structural motif that makes organic molecules highly applicable as reagents and catalysts in various organic transformations ${ }^{1-3}$ and pharmacologically potent compounds in biological systems. ${ }^{4-7}$ Several research papers and reviews reveal this undeniable potential and prove that the search for simple protocols to synthesize diselenides is a highly desirable. Considering their usage in organic synthesis diselenides possess several advantages - they are stable and can be easily converted to electrophilic, nucleophilic and radical reagents. ${ }^{8-24}$

The biological potential of diaryl diselenides is mainly associated with their GPx-like activity. Glutathione peroxidase (GPx) is a selenoenzyme that, based on the presence of catalytically active aminoacid selenocysteine ( $\mathrm{Sec}, \mathrm{U})$, eliminates reactive oxygen and nitrogen species enabling to maintain the redox homeostasis inside the cell. ${ }^{25,26}$ It is assumed that the most-studied GPx mimic ebselen ( $N$ phenylbenzisoselenazo-3(2H)-one, 1), through the reaction with thiols, can be transformed to the corresponding diaryl diselenide with amido function $\mathbf{2}$ and in this form act as a bio-active intermediate that eliminates peroxides. ${ }^{27-29}$

It has been proven that Se-N bond is not necessary to observe an enhanced GPx-like activity. Non-bonding selenium-heteroatom interactions can increase the antioxidant capacity what was established in the case of several diaryl diselenides possessing an additional heteroatom. Examples of highly reactive molecules 3-7 are presented in Scheme 1. ${ }^{30-32}$

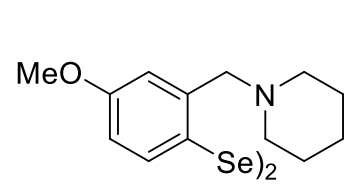

3

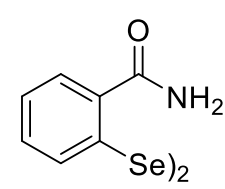

5

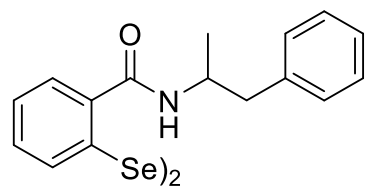

4<smiles>O=S(=O)(O)c1ccccc1C1=NCCO1</smiles>

6

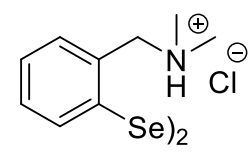

7

Scheme 1. Examples of diaryl diselenides with enhanced GPx-like activity.

Currently, we can specify few methodologies to synthesize $N$-substituted diselenides 8 . Direct reduction of benzisoselenazolones with different reducing agents - sodium borohydride, hydrazine, ascorbate or phosphoric acid (h), ${ }^{33-36}$ nucleophilic substitution of 0 -iodobenzamides with lithium diselenide (i), ${ }^{37}$ under radical conditions from the reaction of 2-benzylselenobenzamides with triphenyltin hydride and further treatment with benzoyl peroxide $(\mathbf{j})^{38}$ and few protocols starting from 2,2-diselenobis(benzoic acid) - through the formation of the corresponding dichloride and further reaction with amine $(\mathbf{k})^{39}$ or by EDC (I) ${ }^{40}$ and DCCmediated $(\mathbf{m})^{41}$ coupling reactions (Scheme 2 ). 


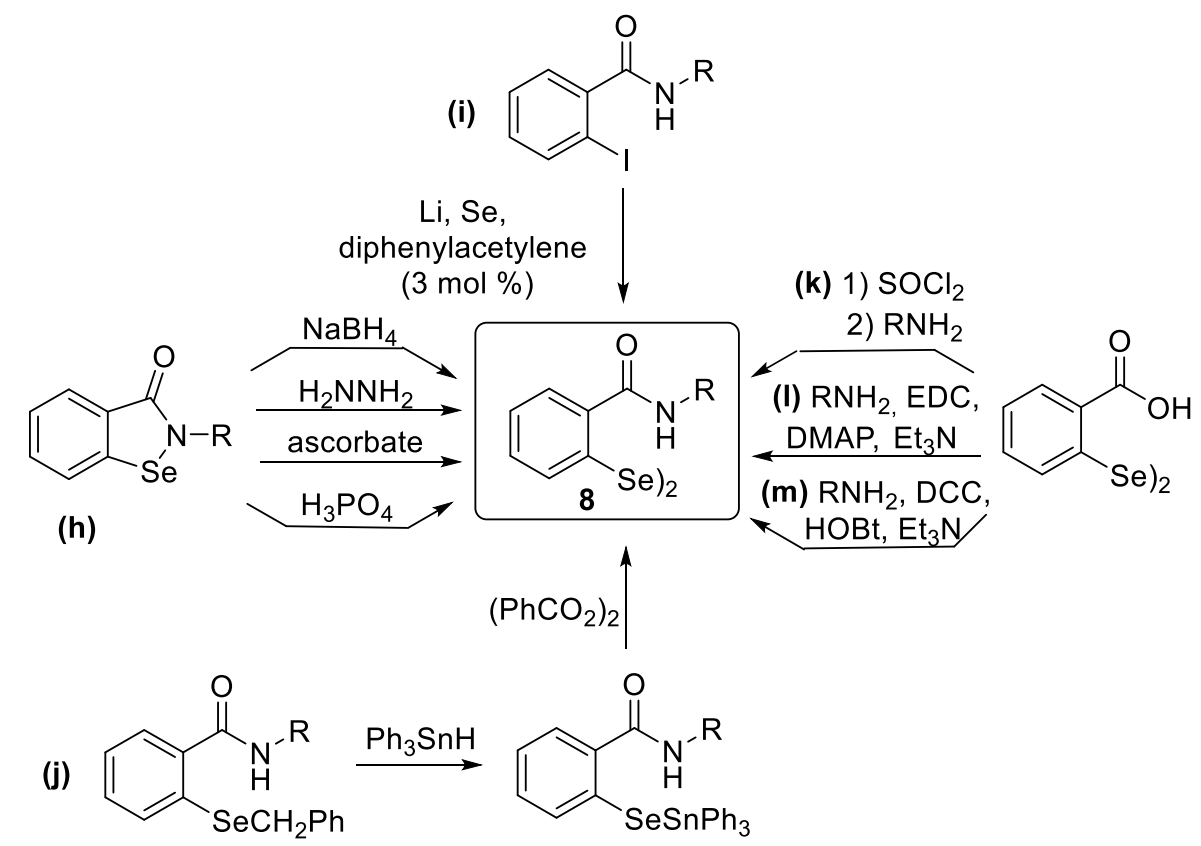

Scheme 2. Approaches to diselenides with amido function $\mathbf{8}$.

The aim of this study was the synthesis of diaryl diselenides with an amido function substituted on the nitrogen atom by a phenyl ring carrying electron withdrawing or electron donating groups and comparison of their antioxidant activity to the GPx-capacity of the corresponding benzisoselenazolones and ebselen.

\section{Results and Discussion}

First, a series of benzisoselenazolones 1, 9-14 was prepared by two procedures, from 2,2-diselenobis(benzoic acid) (method $A)^{39}$ and by our method $B{ }^{42-44}$ involving the treatment of $N$-substituted $O$-iodobenzamide with dilithium diselenide, providing significantly better yields (Scheme 3).

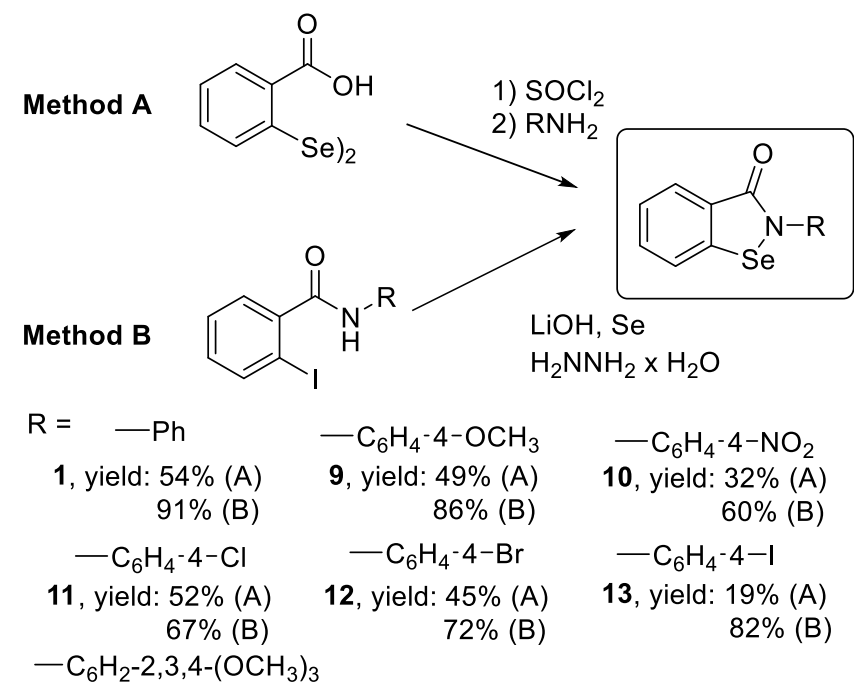

14, yield: $55 \%(\mathrm{~A})$

$75 \%(B)$

Scheme 3. Synthesized benzisoselenazolones 1, 9-14. 
Compounds 1, 9-14 were further transformed into the corresponding diselenides 2, 15-20 using sodium borohydride as the reducing agent followed by air oxidation (method $\mathrm{C}$ ), however, low yields required further studies. During our investigations on the synthesis of ebselen derivatives, based on the reaction of $N$ substituted $o$-iodobenzamide with dilithium diselenide, we observed that water influences the reaction course leading to the formation of diselenides as by-products. Consequently, a new one-step synthesis of compounds 2, 15-20 was developed (method D). According to our previously proposed mechanism for the formation of ebselen derivatives, in the first reaction step corresponding diselenides are formed, which are then oxidized to benzisoselenazolones. ${ }^{42}$ Apparently, the addition of a small amount of water (5\%) decreases the solubility of diselenides what prevents further oxidation reactions. Increasing the amount of added water completely inhibited the product formation and the unreacted substrate was isolated after $24 \mathrm{~h}$. Although the newly presented procedure is based on the known reaction of $o$-iodobenzamides with LiSeSeLi (method i) significant improvements have been achieved. Lithium diselenide is obtained by a modified protocol from selenium and lithium hydroxide in the presence of hydrazine hydrate, and no catalyst is needed for the reaction to proceed. ${ }^{42}$ Formation of monoselenides in the reaction mixture was not observed. ${ }^{37}$ Addition of water (5\%) to the reaction medium enables to obtain diaryl diselenides as the only products directly from $o$-iodobenzamides (Scheme 4).

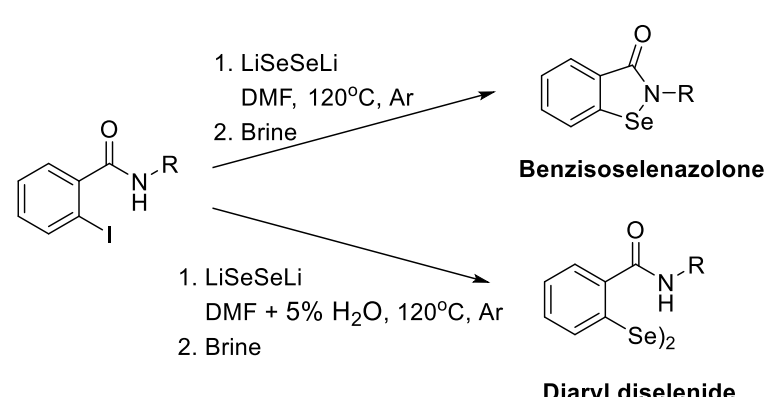

Scheme 4. Water-controlled synthesis of diaryl diselenides.

Results for the synthesis of diaryl diselenides $\mathbf{2 , 1 5 - 2 0}$ by both methods C and D are collected in Table 1. Reducing the procedure to one step increased the overall yields of the reactions. The newly developed waterdependent methodology was significantly more efficient than the synthesis of benzisoselenazolone and its further reduction and air oxidation.

All of the obtained benzisoselenazolones and diselenides were evaluated as in vitro antioxidants using the NMR assay proposed by Iwaoka. ${ }^{45}$ Time of conversion of dithiol (DTT ${ }^{\text {red }}$ ) to a disulphide (DTT ${ }^{\text {ox }}$ ) in the presence of $\mathrm{H}_{2} \mathrm{O}_{2}$ and $10 \%$ of selenocatalyst was measured in selected time intervals (Table 2 ). The highest activity was obtained for benzisoselenazolone $\mathbf{1 4}$ with an additional methoxy moiety. In this case no substrate was observed after $5 \mathrm{~min}$ of the reaction time. 
Table 1. Results of diselenides synthesis according to method C and D

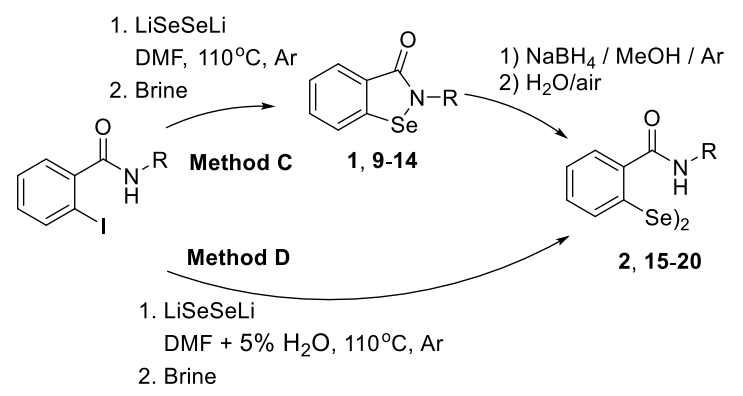

\begin{tabular}{cccc}
\hline $\mathrm{R}=$ & Compound & Method C [\%] & Method D [\%] \\
\hline$-\mathrm{Ph}$ & $\mathbf{2}$ & 34 & 42 \\
$-\mathrm{C}_{6} \mathrm{H}_{4}-4-\mathrm{OCH}_{3}$ & 15 & 53 & 70 \\
$-\mathrm{C}_{6} \mathrm{H}_{4}-4-\mathrm{NO}_{2}$ & 16 & 22 & 45 \\
$-\mathrm{C}_{6} \mathrm{H}_{4}-4-\mathrm{Cl}$ & 17 & 24 & 69 \\
$-\mathrm{C}_{6} \mathrm{H}_{4}-4-\mathrm{Br}$ & 18 & 17 & 76 \\
$-\mathrm{C}_{6} \mathrm{H}_{4}-4-\mathrm{I}$ & 19 & 26 & 43 \\
$-\mathrm{C}_{6} \mathrm{H}_{2}-2,3,4-\left(\mathrm{OCH}_{3}\right)_{3}$ & 20 & 22 & 29 \\
\hline
\end{tabular}

Table 2. Results of the in vitro antioxidant activity measurement for benzisoselenazolones and diselenides

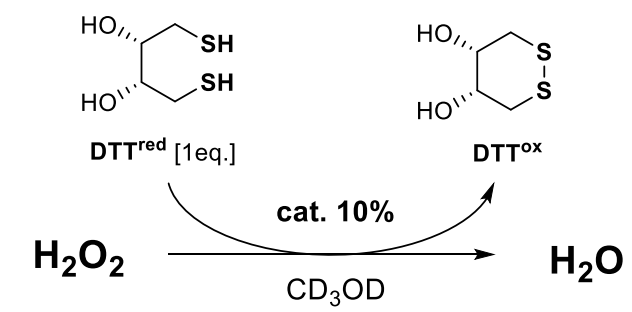

\begin{tabular}{cccccc}
\hline & \multicolumn{5}{c}{ Remaining dithiothreitol [\%] } \\
\hline Catayst & $3 \mathrm{~min}$ & $5 \mathrm{~min}$ & $15 \mathrm{~min}$ & $30 \mathrm{~min}$ & $60 \mathrm{~min}$ \\
\hline $\mathbf{1}$ & 84 & 75 & 64 & 58 & 52 \\
9 & 64 & 41 & 5 & 0 & 0 \\
10 & 25 & 7 & 5 & 0 & 0 \\
11 & 78 & 68 & 50 & 38 & 36 \\
12 & 86 & 83 & 79 & 75 & 71 \\
13 & 42 & 0 & 0 & 0 & 0 \\
14 & 5 & 0 & 0 & 0 & 0 \\
2 & 89 & 83 & 74 & 68 & 63 \\
15 & 84 & 73 & 63 & 57 & 52 \\
16 & 79 & 58 & 10 & 0 & 0 \\
17 & 61 & 53 & 26 & 19 & 12 \\
18 & 81 & 72 & 55 & 41 & 23 \\
19 & 89 & 83 & 81 & 78 & 74 \\
20 & 96 & 82 & 41 & 6 & 0 \\
\hline
\end{tabular}


All derivatives were also tested as cytotoxic agents towards prostate cancer cell line DU145. Results are collected in Table 3. The highest cytotoxic activity against cancer cells was observed for diselenides bearing additional methoxy substituents in the $N$-phenyl ring 15 and 20. Compounds exhibiting cytotoxic potential were additionally tested on normal cell line PNT1A. The data showed that both compounds demonstrated low toxicity towards PNT1A cells. They were also more active than commercially used cisplatin. Conversely, compounds $\mathbf{9}$ and $\mathbf{2}$ exhibited the same cytotoxic activity against noncancerous and cancer cells.

Table 3. Cytotoxic activity of benzisoselenazolones and diselenides expressed as IC50. Values ( $\mu \mathrm{M})$ are means \pm SD

\begin{tabular}{cccc}
\hline Entry & Compound & DU-145 & PNT1A \\
\hline 1 & 1 & $21.16 \pm 0.60$ & $30.57 \pm 0.12$ \\
2 & 9 & $19.80 \pm 13$ & $20.35 \pm 0.08$ \\
3 & 10 & $>40$ & - \\
4 & 11 & $>40$ & - \\
5 & 12 & $>40$ & - \\
6 & 13 & $>40$ & - \\
7 & 14 & $20.84 \pm 0.37$ & $>40$ \\
8 & 2 & $20.70 \pm 0.28$ & $19.76 \pm 0.14$ \\
9 & 15 & $11.24 \pm 0.97$ & $>40$ \\
10 & 16 & $>40$ & - \\
11 & 17 & $>40$ & - \\
12 & 18 & $>40$ & - \\
13 & 19 & $>40$ & - \\
14 & 20 & $11.12 \pm 0.99$ & $>40$ \\
15 & Cisplatin & $\mathbf{2 4 . 1 4} \pm \mathbf{0 . 7 9}$ & - \\
\hline
\end{tabular}

\section{Conclusions}

Herein, we have established that by adding $5 \%$ of water we can initiate the formation of a Se-Se instead of Se$\mathrm{N}$ bond. Simple modification of the previously presented methodology to synthesize benzisoselenazolones led to the development of an efficient protocol to obtain diaryl diselenides with amido function. A series of diselenides was synthesized by two methods: from the reduction and further air oxidation of previously obtained benzisoselenazolones and by the newly developed methodology which was more efficient. An additional advantage of the new methodology is the lack of monoselenides as the side products. All compounds were tested as in vitro antioxidants and cytotoxic agents. The highest in vitro antioxidant activity was obtained for benzisoselenazolone $\mathbf{1 4}$ for which no substrate was observed after 5 min. The cytotoxic activity assay performed on prostate cancer cell line DU145 showed that the presence of the methoxy substituent, also attached to the $N$-phenyl ring of compound $\mathbf{1 4}$, influences the biological activity of derivatives $\mathbf{1 5}$ and $\mathbf{2 0}$ with the highest cytotoxic potential. It is important to note that both compounds $\mathbf{1 5}$ and 20 showed specific activity against cancer cells higher than cisplatin but not against normal epithelial cell line PNT1A. Thus, it can be concluded that both compounds are worth of further study as potential drugs. 


\section{Experimental Section}

General. ${ }^{1} \mathrm{H}$ NMR spectra were obtained at 400 or $700 \mathrm{MHz}$ and chemical shifts were recorded relative to $\mathrm{SiMe}_{4}(\delta 0.00)$ or solvent resonance $\left(\mathrm{CDCl}_{3} \delta 7.26, \mathrm{CD}_{3} \mathrm{OD} \delta 3.31\right)$. Multiplicities were given as: $\mathrm{s}$ (singlet), $\mathrm{d}$ (doublet), dd (double doublet), ddd (double double doublet), t (triplet), td (triple doublet), dt (double triplet) and $m$ (multiplet). The number of protons $(n)$ for a given resonance was indicated by $\mathrm{nH}$. Coupling constants were reported as a $\mathrm{J}$ value in $\mathrm{Hz} .{ }^{13} \mathrm{C}$ NMR spectra were acquired at $100.6 \mathrm{MHz}$ and chemical shifts were recorded relative to solvent resonance $\left(\mathrm{CDCl}_{3}\right.$ 877.25). NMR spectra were carried out using $A C D / N M R$ Processor Academic Edition. Commercially available solvents DMF, DCM and MeOH (Aldrich) and chemicals were used without further purification. Column chromatography was performed using Merck 40-63D 60A silica gel.

\section{Synthesis of compounds $1,9-14$}

Method A. ${ }^{44}$ Preparation of 2-(chloroseleno)benzoyl chloride. To a suspension of selenium (12.7 mmol) in water $(7 \mathrm{~mL})$ sodium borohydride $(25.3 \mathrm{mmol})$ was added under argon atmosphere. Mixture was stirred for $0.5 \mathrm{~h}$ at $0{ }^{\circ} \mathrm{C}$, selenium $(12.7 \mathrm{mmol})$ was added, and stirring was continued at the same temperature for $1 \mathrm{~h}$. Mixture was warmed to room temperature and stirred for $18 \mathrm{~h}$. After adding $40 \% \mathrm{NaOH}(5 \mathrm{~mL})$ the reaction was cooled to $5{ }^{\circ} \mathrm{C}$ and the diazonium salt of anthranilic acid was added dropwise. (The diazonium salt was prepared $z$ in advance - to a solution of anthranilic acid $(26.0 \mathrm{mmol})$ and concentrated hydrochloric acid (5 $\mathrm{mL})$ in water $(15 \mathrm{~mL})$ cooled to $5{ }^{\circ} \mathrm{C}$ a cooled solution of sodium nitrite $(27.5 \mathrm{mmol})$ in water $(15 \mathrm{~mL})$ was added dropwise and the reaction was stirred for $15 \mathrm{~min}$ at $5^{\circ} \mathrm{C}$ ). The mixture was stirred for $3 \mathrm{~h}$ at $60{ }^{\circ} \mathrm{C}$ and for $18 \mathrm{~h}$ at room temperature. The formed precipitate was filtered off and the solution was acidified to $\mathrm{pH}=1$ by $36 \% \mathrm{HCl}$. The formed precipitate was filtrated. The crude product was purified by washing with boiling water and dried in air. Acid $(10.0 \mathrm{mmol})$ was further converted to 2-(chloroseleno)benzoyl chloride by heating with thionyl chloride $(20 \mathrm{~mL})$ at $85{ }^{\circ} \mathrm{C}$ for $3 \mathrm{~h}$. Thionyl chloride was distilled off, and the crude product was used without further purification.

Preparation of benzisoselenazlones. To a solution of amine $(2.0 \mathrm{mmol})$ and triethylamine $(4.0 \mathrm{mmol})$ in dichloromethane 2-(chloroseleno)benzoyl chloride $(1.0 \mathrm{mmol})$ was added. The mixture was stirred for $24 \mathrm{~h}$ at room temperature, poured on water and extracted with DCM. The combined organic layers were dried over anhydrous magnesium sulfate and evaporated. Yields: 54\% 1, 49\% 9, 32\% 10, 52\% 11, 45\% 12, 19\% 13, 55\% 14.

Method B. ${ }^{42}$ Hydrazine hydrate $(8.0 \mathrm{mmol})$ was added dropwise to the mixture of selenium powder (1.2 $\mathrm{mmol}$ ) and lithium hydroxide $(3.6 \mathrm{mmol})$ in DMF $(3 \mathrm{~mL})$. Reaction was heated to $120^{\circ} \mathrm{C}$ and stirred for $15 \mathrm{~min}$ under argon atmosphere. After cooling to room temperature the amide $(1.0 \mathrm{mmol})$ in DMF ( $2 \mathrm{~mL})$ was added. The reaction mixture was heated to $120^{\circ} \mathrm{C}$ and stirred for $20 \mathrm{~h}$ under argon atmosphere. The solution was cooled, $25 \mathrm{~mL}$ of brine was added and the mixture was stirred for additional 20h. Precipitate was filtered under vacuum, washed with water and dried in air. The crude product was purified by column chromatography (silica gel, DCM:MeOH 99.2 : 0.8). Yields: 91\% 1, 86\% 9, 60\% 10, 67\% 11, 72\% 12, 82\% 13, $75 \% 14$.

N-(4-Chlorophenyl)-1,2-benzisoselenazol-3(2H)-one (11). ${ }^{33}$ Yield: Method A: 52\%, Method B: 67\%, mp 179$181{ }^{\circ} \mathrm{C}\left(\mathrm{mp} \mathrm{181-181.5}{ }^{\circ} \mathrm{C}\right),{ }^{1} \mathrm{H}$ NMR (700 MHz, DMSO) $\delta=7.51\left(\mathrm{t}, J 7.0 \mathrm{~Hz}, 1 \mathrm{H}_{\mathrm{ar}}\right), 7.54(\mathrm{~d}, \mathrm{~J}=8.4 \mathrm{~Hz}, 2 \mathrm{Har}), 7.71-$ $7.73\left(\mathrm{~m}, 3 \mathrm{H}_{\mathrm{ar}}\right), 7.93\left(\mathrm{~d}, J 6.3 \mathrm{~Hz}, 1 \mathrm{H}_{\mathrm{ar}}\right), 8.11\left(\mathrm{~d}, J 8.4 \mathrm{~Hz}, 1 \mathrm{H}_{\mathrm{ar}}\right) \mathrm{ppm} ;{ }^{13} \mathrm{C} \mathrm{NMR}\left(100.6 \mathrm{MHz}, \mathrm{CDCl}_{3}\right) \delta=124.73$ 
$\left(\mathrm{CH}_{\mathrm{ar}}\right), 127.53\left(2 \times \mathrm{CH}_{\mathrm{ar}}\right), 127.72\left(\mathrm{CH}_{\mathrm{ar}}\right), 128.24(\mathrm{Car}), 130.42\left(2 \times \mathrm{CH}_{\mathrm{ar}}\right), 130.49\left(\mathrm{CH}_{\mathrm{ar}}\right), 133.15\left(\mathrm{C}_{\mathrm{ar}}\right), 133.77\left(\mathrm{CH}_{\mathrm{ar}}\right)$, $138.33\left(\mathrm{C}_{\mathrm{ar}}\right), 138.70\left(\mathrm{C}_{\mathrm{ar}}\right), 166.72(\mathrm{C}=\mathrm{O}) \mathrm{ppm} ;{ }^{77} \mathrm{Se}\left(76.3 \mathrm{MHz}, \mathrm{CDCl}_{3}\right), \delta=967.61 \mathrm{ppm}, \mathrm{IR}$ 1649, 1631, 1600, $1584,1527,1505,1487,1448,1432,1409,1381,1348,1309,1274,1232,1198,1128,1069,1025 \mathrm{~cm}^{-1}$.

\section{Synthesis of diselenides 2, 15-20}

Method C. To a solution of benzisoselenazolone $1,9-14(1.0 \mathrm{mmol})$ in methanol $(10 \mathrm{~mL})$ cooled to $0^{\circ} \mathrm{C}$, sodium borohydride $(1.0 \mathrm{mmol})$ was added and the mixture was stirred for $1 \mathrm{~h}$. Water $(15 \mathrm{~mL})$ was added and the mixture was oxidized with air for $1 \mathrm{~h}$. Formed precipitate was filtered and dried in air.

Method D. Selenium powder $(1.2 \mathrm{mmol})$ and lithium hydroxide $(3.6 \mathrm{mmol})$ were weight into a single neck flask under argon atmosphere and dissolved in a mixture of DMF $(2.85 \mathrm{~mL})$ and water $(0.15 \mathrm{~mL})$. Hydrazine hydrate $(0.8 \mathrm{mmol})$ was added dropwise and the mixture was heated to $120{ }^{\circ} \mathrm{C}$ and stirred for 15 minutes. After cooling to room temperature the amide $(1.0 \mathrm{mmol})$ dissolved in DMF $(2 \mathrm{~mL})$ was added. The reaction mixture was heated to $120{ }^{\circ} \mathrm{C}$ and stirred for $20 \mathrm{~h}$ under argon atmosphere. The solution was cooled to room temperature, $25 \mathrm{~mL}$ of brine were added, and acidified with hydrochloric acid to $\mathrm{pH}=5$. The mixture was stirred for $1 \mathrm{~h}$ at room temperature. The obtained precipitate was filtered under vacuum, washed with water and dried in air. The crude product was purified by multiple rinsing with boiling DCM.

2,2'-Diselenobis( $N$-phenylbenzamide) (2) ${ }^{\mathbf{4 2}}$ Yield: Method C: 34\%, Method D: 42\%, mp 259-261 ${ }^{\circ} \mathrm{C}$ (lit. ${ }^{46} \mathrm{mp}$ 256-257 $\left.{ }^{\circ} \mathrm{C}\right),{ }^{1} \mathrm{H}$ NMR $(700 \mathrm{MHz}, \mathrm{DMSO}) \delta=7.15\left(\mathrm{t}, J 7.0 \mathrm{~Hz}, 2 \mathrm{H}_{\mathrm{ar}}\right), 7.36-7.43\left(\mathrm{~m}, 6 \mathrm{H}_{\mathrm{ar}}\right), 7.45(\mathrm{td}, J 1.4,7.0 \mathrm{~Hz}$, $\left.2 \mathrm{H}_{\mathrm{ar}}\right), 7.76\left(\mathrm{~d}, J 7.7 \mathrm{~Hz}, 4 \mathrm{H}_{\mathrm{ar}}\right), 7.78$ (dd, J 1.4, 8.4 Hz, $\left.2 \mathrm{H}_{\mathrm{ar}}\right), 7.96$ (d, J 7.7 Hz, 2Har), $10.55(\mathrm{~s}, 2 \mathrm{H}, 2 \mathrm{xNH}) \mathrm{ppm} ;{ }^{13} \mathrm{C}$ NMR (100.6 MHz, DMSO) $\delta 120.66\left(4 \times \mathrm{CH}_{\mathrm{ar}}\right), 124.24\left(2 \times \mathrm{CH}_{\mathrm{ar}}\right), 126.52\left(2 \times \mathrm{CH}_{\mathrm{ar}}\right), 128.73\left(2 \times \mathrm{C}_{\mathrm{ar}}\right), 128.82\left(4 \times \mathrm{CH}_{\mathrm{ar}}\right)$, $130.27\left(2 \times \mathrm{C}_{\mathrm{ar}}\right), 132.06\left(2 \mathrm{xCH}_{\mathrm{ar}}\right), 132.13\left(2 \times \mathrm{C}_{\mathrm{ar}}\right), 133.88\left(2 \mathrm{xCH}_{\mathrm{ar}}\right), 138.73\left(2 \mathrm{xCH}_{\mathrm{ar}}\right), 166.43(2 \times \mathrm{C}=0) \mathrm{ppm} ;{ }^{77} \mathrm{Se}$ (76.3 MHz, DMSO), $\delta=443.67$ ppm, IR 3291, 1666, 1636, 1596, 1581, 1559, 1520, 1499, 1457, 1434, 1322, $1290,1255,1176,1154,1133,1107,1075,1044,1026 \mathrm{~cm}^{-1}$.

2,2-Diselenobis[N-(4-metoxyphenyl)benzamide] (15) ${ }^{47}$ Yield: Method C: $53 \%$, Method D: $70 \%, \mathrm{mp} 287-289{ }^{\circ} \mathrm{C}$ (mp 290-292 $\left.{ }^{\circ} \mathrm{C}\right),{ }^{1} \mathrm{H}$ NMR (700 MHz, DMSO) $\delta 3.75\left(\mathrm{~s}, 6 \mathrm{H}, 2 \mathrm{xOCH}_{3}\right), 6.96\left(\mathrm{~d}, J 9.1 \mathrm{~Hz}, 4 \mathrm{H}_{\mathrm{ar}}\right), 7.39(\mathrm{t}, J 7.0 \mathrm{~Hz}$, $2 \mathrm{H}_{\mathrm{ar}}$ ), $7.44\left(\mathrm{t}, J 7.0 \mathrm{~Hz}, 2 \mathrm{H}_{\mathrm{ar}}\right), 7.67\left(\mathrm{~d}, J 9.1 \mathrm{~Hz}, 4 \mathrm{H}_{\mathrm{ar}}\right), 7.77\left(\mathrm{~d}, J 8.4 \mathrm{~Hz}, 2 \mathrm{H}_{\mathrm{ar}}\right), 7.93$ (d, J $\left.7.7 \mathrm{~Hz}, 2 \mathrm{H}_{\mathrm{ar}}\right), 10.42(\mathrm{~s}, 2 \mathrm{H}$, $2 x N H) p p m ;{ }^{13} \mathrm{C} \mathrm{NMR}\left(100.6 \mathrm{MHz}, \mathrm{DMSO}-d_{6}\right) \delta 55.70\left(2 \mathrm{xOCH}_{3}\right), 114.33\left(4 \mathrm{xCH}_{\mathrm{ar}}\right), 122.66\left(4 \times \mathrm{CH}_{\mathrm{ar}}\right), 126.84$ $\left(2 \times \mathrm{CH}_{\mathrm{ar}}\right), 128.92\left(2 \times \mathrm{CH}_{\mathrm{ar}}\right), 130.62\left(2 \times \mathrm{CH}_{\mathrm{ar}}\right), 132.15\left(2 \times \mathrm{CH}_{\mathrm{ar}}\right), 132.35\left(2 \times \mathrm{C}_{\mathrm{ar}}\right), 132.43\left(2 \times \mathrm{C}_{\mathrm{ar}}\right), 134.30\left(2 \times \mathrm{C}_{\mathrm{ar}}\right)$, $156.37\left(2 \times C_{a r}\right), 166.41(2 x C=0)$ ppm; ${ }^{77} \mathrm{Se}\left(76.3 \mathrm{MHz}, \mathrm{CDCl}_{3}\right), \delta 443.49 \mathrm{ppm}, \mathrm{IR} 1631,1510,1410,1247,1027$ $\mathrm{cm}^{-1}$.

2,2'-Diselenobis[ $N$-(4-nitrophenyl)benzamide] (16) ${ }^{47}$ Yield: Method C: $22 \%$, Method D: 45\%, mp 254-256 ${ }^{\circ} \mathrm{C}$, ${ }^{1} \mathrm{H}$ NMR (700 MHz, DMSO) $\delta$ 7.44-7.52 (m, 4Har), $7.82\left(\mathrm{~d}, J 7.2 \mathrm{~Hz}, 2 \mathrm{H}_{\mathrm{ar}}\right), 8.02\left(\mathrm{~d}, J 7.2 \mathrm{~Hz}, 2 \mathrm{H}_{\mathrm{ar}}\right), 8.06$ (d, J 9.6 $\left.\mathrm{Hz}, 4 \mathrm{H}_{\mathrm{ar}}\right), 8.31\left(\mathrm{~d}, J 9.6 \mathrm{~Hz}, 4 \mathrm{H}_{\mathrm{ar}}\right), 11.09$ (s, 2H, 2xNH) ppm; ${ }^{13} \mathrm{C} \mathrm{NMR}(100.6 \mathrm{MHz}, \mathrm{DMSO}) \delta 120.7\left(4 \times \mathrm{CH}_{\mathrm{ar}}\right), 125.3$ $\left(4 \mathrm{xCH}_{\mathrm{ar}}\right), 127.1\left(2 \times \mathrm{CH}_{\mathrm{ar}}\right), 129.6\left(2 \times \mathrm{CH}_{\mathrm{ar}}\right), 130.9\left(2 \times \mathrm{C}_{\mathrm{ar}}\right), 132.8\left(2 \times \mathrm{Car}_{\mathrm{ar}}\right), 133.1\left(2 \times \mathrm{CH}_{\mathrm{ar}}\right), 133.6\left(2 \times \mathrm{C}_{\mathrm{ar}}\right), 143.3$ $\left(2 x \mathrm{CH}_{\mathrm{ar}}\right), 145.4\left(2 \mathrm{xC}_{\mathrm{ar}}\right), 167.4(2 \mathrm{xC}=\mathrm{O}) \mathrm{ppm} ;{ }^{77} \mathrm{Se}$ (76.3 MHz, DMSO), $\delta 447.41 \mathrm{ppm}, \mathrm{IR} 3392,1660,1610,1595$, $1583,1535,1493,1430,1403,1328,1302,1280,1237,1177,1138,1110,1094,1055,1026 \mathrm{~cm}^{-1}$.

2,2'-Diselenobis[ $\mathbf{N}$-(4-chlorophenyl)benzamide] (17) ${ }^{33}$ Yield: Method C: $24 \%$, Method D: 69\%, ${ }^{1} \mathrm{H}$ NMR (700 $\mathrm{MHz}, \mathrm{DMSO}) \delta=7.45(\mathrm{dt}, J 0.7,7.0 \mathrm{~Hz}, 2 \mathrm{H} \mathrm{ar}), 7.47-7.51\left(\mathrm{~m}, 6 \mathrm{H}_{\mathrm{ar}}\right), 7.81(\mathrm{dd}, J 1.4,8.4 \mathrm{~Hz}, 2 \mathrm{Har})$, ), $7.84(\mathrm{~d}, J 9.1$ $\left.\mathrm{Hz}, 4 \mathrm{H}_{\mathrm{ar}}\right), 7.98$ (dd, J 1.4, 7.7 Hz, $\left.2 \mathrm{H}_{\mathrm{ar}}\right), 10.69(\mathrm{~s}, 2 \mathrm{H}, 2 \mathrm{xNH}) \mathrm{ppm} ;{ }^{13} \mathrm{C} \mathrm{NMR}(100.6 \mathrm{MHz}, \mathrm{DMSO}) \delta 123.6\left(4 \times \mathrm{CH}_{\mathrm{ar}}\right)$, $127.6\left(2 \times \mathrm{C}_{\mathrm{ar}}\right), 128.0\left(2 \times \mathrm{CH}_{\mathrm{ar}}\right), 129.3\left(2 \times \mathrm{C}_{\mathrm{ar}}\right), 130.1\left(4 \mathrm{xCH}_{\mathrm{ar}}\right), 130.5\left(2 \times \mathrm{CH}_{\mathrm{ar}}\right), 131.7\left(2 \times \mathrm{C}_{\mathrm{ar}}\right), 133.5\left(2 \times \mathrm{CH}_{\mathrm{ar}}\right), 135.0$ $\left(2 \times \mathrm{C}_{\mathrm{ar}}\right), 139.0\left(2 \times \mathrm{CH}_{\mathrm{ar}}\right), 167.9(2 \times \mathrm{C}=\mathrm{O}) \mathrm{ppm} ;{ }^{77} \mathrm{Se}$ (76.3 MHz, DMSO), $\delta 444.45 \mathrm{ppm}, \mathrm{IR} 3283,1641,1593,1512$, $1396,1317,1252,1096,1015 \mathrm{~cm}^{-1}$.

2,2-Diselenobis[N-(4-bromophenyl)benzamide] (18) ${ }^{\mathbf{4 8}}$ Yield: Method C: $17 \%$, Method D: $76 \%, \mathrm{mp} 248-250{ }^{\circ} \mathrm{C}$, ${ }^{1} \mathrm{H}$ NMR $(700 \mathrm{MHz}, \mathrm{DMSO}){ }^{1} \mathrm{H}$ NMR $\left(700 \mathrm{MHz}, \mathrm{DMSO}-d_{6}\right) \delta 7.42\left(\mathrm{dt}, J 0.7,7.0 \mathrm{~Hz}, 2 \mathrm{H}_{\mathrm{ar}}\right), 7.46(\mathrm{dt}, J 1.4,7.7 \mathrm{~Hz}$, 
$\left.2 \mathrm{H}_{\mathrm{ar}}\right), 7.58\left(\mathrm{~d}, J 8.4 \mathrm{~Hz}, 4 \mathrm{H}_{\mathrm{ar}}\right), 7.75\left(\mathrm{~d}, J 9.1 \mathrm{~Hz}, 4 \mathrm{H}_{\mathrm{ar}}\right), 7.78(\mathrm{dd}, J$ 0.7, 8.4 Hz, 2Har), $7.96(\mathrm{dd}, J 0.7,7.0 \mathrm{~Hz}, 2 \mathrm{Har})$, 10.67 (s, 2H, 2xNH) ppm; ${ }^{13} \mathrm{C}$ NMR (100.6 MHz, DMSO) $\delta 116.36\left(2 x \mathrm{CH}_{\mathrm{ar}}\right), 122.92\left(4 \times \mathrm{CH}_{\mathrm{ar}}\right), 126.96\left(2 \times \mathrm{CH}_{\mathrm{ar}}\right)$, $129.20\left(2 \times C_{a r}\right), 130.74\left(2 \times C_{a r}\right), 132.05\left(4 \times C_{a r}\right), 132.52\left(2 \times C_{a r}\right), 132.69\left(2 \times C_{a r}\right), 134.00\left(2 \times C_{a r}\right), 138.54\left(2 \times C_{a r}\right)$, 166.88 (2xC=O) ppm; ${ }^{77}$ Se (76.3 MHz, DMSO), $\delta 445.26$ ppm, IR 1643, 1587, 1509, 1489, 1471, 1455, 1425, $1391,1314,1286,1251,1237,1097,1073,1045,1025,1011 \mathrm{~cm}^{-1}$.

2,2-Diselenobis[N-(4-jodophenyl)benzamide] (19) ${ }^{33}$ Yield: Method C: $26 \%$, Method D: 46\%, mp $260-264{ }^{\circ} \mathrm{C},{ }^{1} \mathrm{H}$ NMR (700 MHz, DMSO) $\delta 7.41$ (dt, J 1.4, 7.7 Hz, 2Har), $7.46\left(\mathrm{dt}, J 1.4,7.7 \mathrm{~Hz}, 2 \mathrm{H}_{\mathrm{ar}}\right), 7.62\left(\mathrm{~d}, J 8.4 \mathrm{~Hz}, 4 \mathrm{H}_{\mathrm{ar}}\right), 7.72$ (d, J 8.4 Hz, 4Har), 7.78 (dd, J 0.7, 7.7 Hz, 2Har), 7.95 (dd, J 0.7, 7.7 Hz, 2Har), $10.63(\mathrm{~s}, 2 \mathrm{H}, 2 \times N H) \mathrm{ppm} ;{ }^{13} \mathrm{C} \mathrm{NMR}$ $\left(100.6 \mathrm{MHz}, \mathrm{DMSO}-d_{6}\right) \delta 88.44\left(2 \times \mathrm{C}_{\mathrm{ar}}\right), 123.18\left(4 \times \mathrm{CH}_{\mathrm{ar}}\right), 126.98\left(2 \mathrm{xCH}_{\mathrm{ar}}\right), 129.16\left(2 \times \mathrm{CH}_{\mathrm{ar}}\right), 130.75\left(2 \times \mathrm{CH}_{\mathrm{ar}}\right)$, $132.49\left(2 \times \mathrm{C}_{\mathrm{ar}}\right), 132.66\left(2 \times \mathrm{CH}_{\mathrm{ar}}\right), 134.04\left(2 \times \mathrm{C}_{\mathrm{ar}}\right), 137.88\left(4 \times \mathrm{CH}_{\mathrm{ar}}\right), 138.97(2 \times \mathrm{Car}), 166.89(2 \times \mathrm{C}=\mathrm{O}) \mathrm{ppm} ;{ }^{77} \mathrm{Se}(76.3$ $\left.\mathrm{MHz}, \mathrm{DMSO}-d_{6}\right), \delta 445.49$ ppm, IR 3260, 1636, 1597, 1584, 1508, 1456, 1429, 1409, 1391, 1349, 1316, 1232, $1126,1061,1027,1004 \mathrm{~cm}^{-1}$.

2,2-Diselenobis[N-(3,4,5-trimetoxyphenyl)benzamide] (20) ${ }^{49}$ Yield: Method C: $22 \%$, Method D: $29 \%$, mp 156$160{ }^{\circ} \mathrm{C},{ }^{1} \mathrm{H}$ NMR $\left(700 \mathrm{MHz}, \mathrm{CDCl}_{3}\right) \delta 3.87\left(\mathrm{~s}, 6 \mathrm{H}, 2 \mathrm{xOCH}_{3}\right), 3.92\left(\mathrm{~s}, 12 \mathrm{H}, 4 \mathrm{xOCH}_{3}\right), 6.96\left(\mathrm{~s}, 4 \mathrm{H}_{\mathrm{ar}}\right), 7.34(\mathrm{t}, J 7.7 \mathrm{~Hz}$, $\left.2 \mathrm{H}_{\mathrm{ar}}\right), 7.38\left(\mathrm{t}, J 7.7 \mathrm{~Hz}, 2 \mathrm{H}_{\mathrm{ar}}\right), 7.67(\mathrm{~d}, J 7.0 \mathrm{~Hz}, 2 \mathrm{H} \mathrm{ar}), 7.80(\mathrm{~s}, 2 \mathrm{H}, 2 \mathrm{xNH}), 7.99\left(\mathrm{~d}, J 7.7 \mathrm{~Hz}, 2 \mathrm{H}\right.$ ar) ppm; ${ }^{13} \mathrm{C} \mathrm{NMR}$ $\left(100.6 \mathrm{MHz}, \mathrm{CDCl}_{3}\right) \delta 57.26\left(4 \mathrm{xOCH}_{3}\right), 62.00\left(2 \mathrm{xOCH}_{3}\right), 99.19\left(4 \mathrm{xCH}_{\mathrm{ar}}\right), 104.62\left(2 \times \mathrm{C}_{\mathrm{ar}}\right), 127.54\left(2 \times \mathrm{CH}_{\mathrm{ar}}\right), 127.76$ $\left(2 \times \mathrm{CH}_{\mathrm{ar}}\right), 130.38\left(2 \times \mathrm{C}_{\mathrm{ar}}\right), 132.95\left(2 \times \mathrm{CH}_{\mathrm{ar}}\right), 133.12\left(2 \times \mathrm{CH}_{\mathrm{ar}}\right), 134.51\left(4 \times \mathrm{C}_{\mathrm{ar}}\right), 134.83\left(2 \times \mathrm{C}_{\mathrm{ar}}\right), 136.33\left(2 \times \mathrm{C}_{\mathrm{ar}}\right), 154.49$ $(2 \times C=0) \mathrm{ppm}^{77} \mathrm{Se}\left(76.3 \mathrm{MHz}, \mathrm{CDCl}_{3}\right), \delta=454.96 \mathrm{ppm}, \mathrm{IR} 3268,2933,2836,1632,1600,1585,1561,1526$, $1506,1488,1450,1410,1384,1311,1284,1233,1199,1128,1071,1045,1025,1008 \mathrm{~cm}^{-1}$. Elemental Anal. Calcd for $\mathrm{C}_{32} \mathrm{H}_{32} \mathrm{~N}_{2} \mathrm{O}_{8} \mathrm{Se}_{2}$ (730.53): $\mathrm{C}, 52.61 ; \mathrm{H}, 4.42$. Found: $\mathrm{C}, 52.26 ; \mathrm{H}, 4.38$.

\section{Evaluation of in vitro antioxidant activity ${ }^{45}$}

To a solution of compounds 1, 2, 9-20 $(0.015 \mathrm{mmol})$ and dithiothreitole DTT red $(0.15 \mathrm{mmol})$ in $1.1 \mathrm{~mL}$ of $\mathrm{CD}_{3} \mathrm{OD}$ $30 \% \mathrm{H}_{2} \mathrm{O}_{2}(0.15 \mathrm{mmol})$ was added. ${ }^{1} \mathrm{H}$ NMR spectra were measuared right after addition of hydrogen peroxide and then in specific time intervals. The concentration of the substrate was determined according to the changes in the integration on the ${ }^{1} \mathrm{H}$ NMR spectra (Table 2).

\section{SRB viability assay ${ }^{50}$}

Cell culture: The prostate cancer cell line DU-145 was purchased from the American Type Culture Collection (ATTC, Manassas, VA). The DU-145 cells were cultured in MEME medium supplemented with $10 \%$ fetal bovine serum, $1 \%$ penicillin/streptomycin, $2 \mathrm{mM}$ glutamine and $1 \mathrm{mM}$ sodium pyruvate at $37{ }^{\circ} \mathrm{C}$. The PNT1A cells were cultured in RPMI 1640 supplemented with serum, L-glutamine and antibiotics. The cells were maintained at $37{ }^{\circ} \mathrm{C}$ in an atmosphere containing $5 \% \mathrm{CO}_{2}$. Stock solutions of $\mathrm{N}$-substituted ebselen derivatives were prepared in $(0,1 \%)$ DMSO.

SRB assay: Cell viability was measured by Sulphorhodamine B (SRB) assay. The cells were grown to subconfluent levels at the certain culture medium and then seeded into 96 -well plates at $6.0 \times 10^{3}$ cells/ well in the final volume of $200 \mu \mathrm{l}$ in the culture medium for $24 \mathrm{~h}$. Then, they were treated with various concentrations $(2,5,5,10,20,30,40 \mu \mathrm{l})$ of $\mathrm{N}$-substituted ebelsen derivatives for the next $24 \mathrm{~h}$. After incubation, the cells were fixed in $20 \%$ trichloroacetic acid for an $1 \mathrm{~h}$. The plates were washed with distilled water and 0,4\% SRB (Sigma Aldrich ) in $1 \%$ acetic acid solution was added to the plates for 15 minutes. The SRB solution was washed with $1 \%$ acetic acid. SRB was then solubilized in $10 \mathrm{mM}$ Trisma-base solution and the absorbance was measured at $570 \mathrm{~nm}$ using an automated microplate reader. The experiments were done in triplates and the $I_{50}$ values were calculated. 


\section{Acknowledgements}

This work was supported by the National Science Centre, Poland, grant no. UMO-2015/17 /B/ NZ7/03058.

\section{References}

1. Organoselenium Chemistry: A Practical Approach; Back, T. G., Ed.; Oxford University Press: Oxford, 1999.

2. Organoselenium Chemistry; Wirth, T., Ed.; Top. Cur. Chem. 2000. https://doi.org/10.1007/3-540-48171-0

3. Organoselenium Chemistry: Synthesis and Reactions; Wirth, T., Ed.; Wiley-VCH: Weinheim, 2012.

4. Organoselenium Chemistry: Between Synthesis and Biochemistry; Santi, C., Ed.; Bentham, 2014.

5. Pacuła, A. J.; Mangiavacchi, F.; Sancineto, L.; Lenardao, E. J.; Ścianowski, J.; Santi, C. Curr. Chem. Biol., 2015, 9, 97.

\section{https://doi.org/10.2174/2212796810666160120220725}

6. Santi, C.; Marini, F.; Lenardão, E. J. In Organoselenium Compounds in Biology and Medicine Jain, V. K.; Priyadarsini, K. I., Eds.; RSC Pubs.: Croydon, UK, 2017, pp 37-71.

7. Barcellos, A. M.; Abenante, L.; Sarro, M. T.; Leo, I.; Lenardão, E. J.; Perin, G.; Santi, C. Curr. Org. Chem. 2016, 20 , in press.

https://doi.org/10.2174/1385272820666161020162113

8. Ścianowski, J.; Rafiński, Z. In Organoselenium Chemistry: Between Synthesis and Biochemistry; Santi, C., Ed.; Bentham Science Publishers, 2014; pp 8-60.

9. Santi, C.; Santoro, S. In Organoselenium Chemistry Wirth, T., Eds.; Wiley-VCH, 2011; pp 1-51.

10. Santi, C.; Tidei, C. In The Chemistry of Organic Selenium and Tellurium Compounds; Rappoport, Z., Ed.; John Wiley \& Sons, Ltd: Chichester, UK, 2013; Vol. 4, pp 569-655.

11. Wirth, T.; Kulicke, K.J.; Fragale, G. Helv. Chim. Acta 1996, 79, 1957.

https://doi.org/10.1002/hlca.19960790718

12. Santi, C.; Wirth, T. Tetrahedron: Asymmetry 1999, 10, 1019.

https://doi.org/10.1016/S0957-4166(99)00090-7

13. Ścianowski, J.; Rafalski, J.; Banach, A.; Czaplewska, J.; Komoszyńska, A. Tetrahedron: Asymmetry 2013, 24, 1089.

https://doi.org/10.1016/j.tetasy.2013.07.018

14. Rafiński, Z.; Ścianowski, J. Tetrahedron: Asymmetry 2008, 19, 1237.

https://doi.org/10.1016/j.tetasy.2008.04.027

15. Ścianowski, J.; Rafiński, Z.; Wojtczak, A; Burczyński, K. Tetrahedron: Asymmetry 2009, 20, 2871.

https://doi.org/10.1016/j.tetasy.2009.12.001

16. Ścianowski, J. Tetrahedron Lett. 2005, 46, 3334.

https://doi.org/10.1016/j.tetlet.2005.03.073

17. Ścianowski, J.; Rafiński, Z.; Wojtczak, A. Eur. J. Org. Chem. 2006, 14, 3216.

https://doi.org/10.1002/ejoc.200600044

18. Ścianowski, J.; Banach, A.; Uzarewicz-Baig, M.; Wojtczak, A. Eur. J. Org. Chem. 2015, 16, 3477.

19. Rafiński, Z.; Ścianowski, J.; Wojtczak, A. Lett. Org. Chem. 2009, 6, 321.

https://doi.org/10.2174/157017809788489846

20. Ścianowski, J.; Rafiński, Z.; Szuniewicz, A.; Wojtczak, A. Tetrahedron 2009, 65, 10162. 
https://doi.org/10.1016/j.tet.2009.10.005

21. Wirth T. Tetrahedron Lett. 1995, 36, 7849.

https://doi.org/10.1016/0040-4039(95)01668-8

22. Glaubia, S.; Neto, J. S. S.; Pesarico, A.P.; Back, D. F.; Nogueira, C. W.; Zeni, G. Org. Biomol. Chem. 2013, 11, 1199.

https://doi.org/10.1039/c2ob27064a

23. Tomoda, S.; Iwaoka, M. J. Chem. Soc., Chem. Commun. 1988, 1283.

https://doi.org/10.1039/c39880001283

24. Nishibayashi, Y.; Singh, J. D.; Fukuzawa, S. I.; Uemura, S. J. J. Chem. Soc., Perkin Trans. 1 1995, 2871.

https://doi.org/10.1039/p19950002871

25. Mugesh, G.; du Mont, W. W. Chem. Eur. J. 2001, 7, 1365.

https://doi.org/10.1002/1521-3765(20010401)7:7<1365::AID-CHEM1365>3.0.CO;2-Y

26. Roy, G.; Sarma, B. K ; Phadnis, P. P.; Mugesh, G. J. Chem. Sci. 2005, 117, 287.

https://doi.org/10.1007/BF02708441

27. Sarma, K.; Mugesh, G. Chem. Eur. J. 2008, 14, 10603.

https://doi.org/10.1002/chem.200801258

28. Bhabak, K. P.; Mugesh, G. Acc. Chem. Res. 2010, 43, 1408.

https://doi.org/10.1021/ar100059g

29. Orian, L.; Toppo, S. Free Radic. Biol. Med. 2014, 66, 65.

https://doi.org/10.1016/j.freeradbiomed.2013.03.006

30. Prabhu, C. P.; Phadnis, P.P.; Wadawale, A. P.; Priyadarsini, K. I.; Jain, V. K. J. Organomet. Chem. 2012, 713, 42.

https://doi.org/10.1016/j.jorganchem.2012.04.014

31. Soares, L. C.; Alberto, E. E.; Schwab, R. S.; Taube, P. S.; Nascimiento, V.; Rodrigues, O. E. D.; Braga, A. L. Org.

Biomol. Chem. 2012, 10, 6595.

https://doi.org/10.1039/c2ob25539a

32. Bhowmick, D.; Mugesh, G. Tetrahedron 2012, 68, 10550.

https://doi.org/10.1016/j.tet.2012.09.020

33. Młochowski, J.; Kloc, K.; Syper, L.; Inglot, A. D.; Piasecki, E. Liebigs Ann. Chem. 1993, 1239.

https://doi.org/10.1002/jlac.1993199301201

34. Kalai, T.; Mugesh, G.; Roy, G.; Sies, H.; Berente, Z.; Hideg, K. Org. Biomol. Chem. 2005, 3, 3564.

https://doi.org/10.1039/b509865c

35. Satheeshumar, K.; Mugesh, G. Chem. Eur. J. 2011, 17, 4849.

https://doi.org/10.1002/chem.201003417

36. Mbuyi, M.; Christensen, L.; Rensen, M. Bull. Soc. Chim. Belg. 1989, 98, 395.

https://doi.org/10.1002/bscb.19890980607

37. Syper, L.; Młochowski, J. Tetrahedron 1988, 44, 6119.

https://doi.org/10.1016/S0040-4020(01)89801-X

38. Fong, M. C.; Schiesser, C. H. Tertrahedron Lett. 1995, 36, 7329.

https://doi.org/10.1016/0040-4039(95)01511-F

39. Welter, A.; Christiaens, L.; Ferdinand, W. P. Eur. Pat. Appl. EP 44453, 1982, Chem. Abstr., 1982, 96, 199699v.

40. Nascimento, V.; Ferreira, N. L.; Canto, R. F. C.; Schott, K. L.; Walczuk, E. P.; Sancineto, L.; Santi, C.; Rocha, J.

B. T.; Braga, A. L. Eur. J. Med. Chem. 2014, 87, 131. 
https://doi.org/10.1016/i.ejmech.2014.09.022

41. Sancineto, L.; Mariotti, A.; Bagnoli, L.; Marini, F.; Desantis, J.; Iraci, N.; Santi, C.; Pannecouque, Ch.;

Tabarrini, O. J. Med. Chem. 2015, 58, 9601. https://doi.org/10.1021/acs.jmedchem.5b01183

42. Pacuła, A. J.; Ścianowski, J.; Aleksandrzak, K. B. RSC Adv.,2014, 4, 48959. https://doi.org/10.1039/C4RA08631G

43. Pacuła, A. J.; Kaczor, K. B.; Wójtowicz, A.; Antosiewicz, J.; Janecka, A.; Długosz, A.; Janecki, T.; Ścianowski, J. Bioorg. Med. Chem. 2017, 25, 126.

https://doi.org/10.1016/j.bmc.2016.10.018

44. Pacuła, A.J.; Kaczor, K. B.; Antosiewicz, J.; Janecka, A.; Długosz, A.; Janecki, T.; Wojtczak, A.; Ścianowski, J. Molecules 2017, 22, 492.

https://doi.org/10.3390/molecules22030492

45. Kumakura, F.; Mishra, B.; Priyadarsini, K. I.; Iwaoka, M. Eur. J. Org. Chem. 2010, 440.

https://doi.org/10.1002/ejoc.200901114

46. Engman, L.; Hallberg, A. J. Org. Chem. 1989, 54, 2964.

https://doi.org/10.1021/jo00273a03

47. Nattermann, A.CIE GmbH Patent: US4873350 A1, 1989.

48. Bhabak K. P.; Vernekar, A. A.; Jakka, S. R.; Roy, G. Mugesh, G. Org. Biomol. Chem., 2011, 9, 5193. https://doi.org/10.1039/c0ob01234c

49. Griurg, M.; Gołąb, A.; Suchodolski, J.; Kaleta, R.; Krasowska, A.; Piasecki, E.; Piętka-Ottlik, M. Molecules 2017, 22, 974-996.

https://doi.org/10.3390/molecules22060974

50. Mosmann, T. J. Immunol. Methods 1983, 65, 55.

https://doi.org/10.1016/0022-1759(83)90303-4 